\title{
The SNHG16/miR-30a axis promotes breast cancer cell proliferation and invasion by regulating RRM2
}

\author{
S. M. DU \\ Zibo Vocational Institute, Zibo, Shandong, China \\ *Correspondence: dushanmei@126.com
}

Received June 25, 2019 / Accepted September 29, 2019

\begin{abstract}
Long noncoding RNAs (lncRNAs) have been suggested to play vital roles in tumor initiation and progression. Recent studies have reported that the lncRNA small nucleolar RNA host gene 16 (SNHG16) is highly expressed in breast cancer tissue. In the present study, we demonstrated that SNHG16 is an oncogene involved in cell proliferation and invasion in breast cancer. First, we examined the functional role of SNHG16 in breast cancer cells by knocking down SNHG16 expression via siRNA. We found that SNHG16 inhibition reduced the proliferation and invasion of breast cancer cells in vitro. Then, based on bioinformatic prediction and functional assay validation, we demonstrated SNHG16 interaction with miR-30a and its role in breast cancer cells. Finally, we examined the functional role of RRM2 in breast cancer cells by knocking down RRM2 expression via siRNA. Our results indicated that the SNHG16/miR-30a axis regulated the expression of ribonucleotide reductase M2 (RRM2) in breast cancer cells. These results provide novel insight into breast cancer tumorigenesis and suggest that SNHG16 could serve as a therapeutic target in breast cancer.
\end{abstract}

Key words: IncRNA SNHG16, breast cancer, miR-30a, ribonucleotide reductase M2, proliferation, invasion

Breast cancer is one of the most commonly occurring malignant tumors in women, with a recently increased incidence and dramatically decreased onset age $[1,2]$. Despite advances in diagnosis and treatment, the survival rates of breast cancer patients remain low [3]. Abnormal proliferation and invasion by breast cancer cells are major contributors to breast cancer patient death [4]. Therefore, it is of great significance to investigate the mechanisms underlying breast cancer progression.

Long noncoding RNAs (lncRNAs) are defined as a type of RNA that is more than 200 nucleotides in length and has no protein-coding ability [5]. Many studies have shown that $\operatorname{lncRNAs}$ regulate gene expression at the epigenetic, transcriptional and posttranscriptional levels and participate in various physiological processes by interacting with proteins and nucleic acids [6]. Increasing evidence has revealed that dysregulated expression of lncRNAs is involved in tumor initiation, progression, and metastasis [7-10]. In breast cancer, some lncRNAs have been identified as oncogenes or tumor suppressors and have potential use as diagnostic or prognostic markers in breast cancer patients [11, 12].

Although it is commonly accepted that small nucleolar RNA host gene 16 (SNHG16), a noncoding RNA, has upregulated expression and functions as an oncogene that promotes tumorigenesis and tumor progression in multiple cancers [13-16], the regulatory mechanism involving SNHG16 in breast cancer still needs more exploration. In the present study, we explored how SNHG16 contributes to the progression of breast cancer and investigated the underlying mechanisms.

\section{Materials and methods}

Cell culture and transfection. The human breast cancer cell lines MDA-MB-231 and MCF-7 and the immortalized breast epithelial cell line MCF-10A were used in this study. All cells were purchased from the Cell Bank of the Chinese Academy of Sciences (Shanghai, China) and cultured in high-glucose Dulbecco's modified Eagle's medium (DMEM; Thermo Fisher Scientific, MA, USA) supplemented with 10\% fetal bovine serum (FBS; Gibco, CA, USA). All cells were maintained in humidified air at $37^{\circ} \mathrm{C}$ with $5 \% \mathrm{CO}_{2}$.

siRNA against SNHG16 (si-SNHG16), siRNA against RRM2 (si-RRM2), a scrambled negative control siRNA (si-NC), miR-30a mimic, miR-30a inhibitors and the corresponding negative controls (mimic NC and inhibitor NC) were purchased from GenePharma Biotechnology (Shanghai, China). All oligonucleotide sequences are listed in Table 1. 
Lipofectamine 2000 reagents (Invitrogen, Carlsbad, CA, USA) were used to perform all transfections according to the manufacturer's instructions.

RNA isolation and quantitative real-time PCR (qRT-PCR). Total RNA was isolated from tissue samples and cells using RNAiso Plus (TAKARA Biotechnology, Dalian, China) according to the manufacturer's instructions. To measure SNHG16, miR-30a and ribonucleotide reductase M2 (RRM2) mRNA expression, qRT-PCR was performed using a SYBR Green-based PCR Kit (TAKARA Biotechnology). $\beta$-actin or U6 snRNA was used as an internal control. The specific details were described in a previous report [17]. All primers used are listed in Table 2.

Cell proliferation and colony formation assays. The proliferation of breast cancer cells was assessed using a Cell Counting Kit-8 (CCK-8; Beyotime Institute of Biotechnology, Shanghai, China) assay, and the cell colony number was investigated by a colony formation assay. The specific details were described in a previous report [18].

Apoptosis assays. An apoptosis kit (MultiSciences Biotechnology, Hangzhou, China) was used to evaluate apoptosis with a FACSCalibur flow cytometer (Thermo Fisher) according to the manufacturer's instructions. The specific details were described in a previous report [18].

Cell migration and invasion assays. A Transwell assay was performed to investigate the migratory and invasive capabilities of cells using a 24-well Transwell plate (Corning, NY, USA). For the invasion assay, the upper chambers were

Table 1. Oligonucleotide sequences.

\begin{tabular}{ll}
\hline Name & Sequence \\
\hline si-SNHG16 & 5'-GATATCTTAGTCCTAACCATATTGATCCC-3' \\
si-RRM2 & 5'-CGAUGGCAUAGUAAAUGAATT-3' \\
si-NC & 5'-UUCUCCGAACGUGUCACGUTT-3' \\
miR-30a mimic sense & 5'-UGUAAACAUCCUCGA CUGGAAG-3' \\
anti-sense & 5'-UCCAGUCGAGGAUGU UUACAUU-3' \\
mimic NC sense & 5'-UUCUCCGAACGUGUCACGUTT-3' \\
anti-sense & 5'-ACGUGACACGUUCGGAGAATT-3' \\
miR-30a inhibitor & 5'-CUUCCAGUCGAGGAUGUUUACA-3' \\
inhibitor NC & 5'-CAGUACUUUUGUGUAGUACAA-3' \\
\hline
\end{tabular}

Table 2. Sequences of primers used for qRT-PCR.

\begin{tabular}{ll}
\hline Gene & Sequence \\
\hline SNHG16 & F: 5'-CAGAATGCCATGGTTTCCCC-3' \\
& R: 5'-TGGCAAGAGACTTCCTGAGG-3' \\
miR-30a & F: 5'-CGACGACTTTCA-GTCGGATGTT-3' \\
& R: 5'-GTGCAGGGTCCGAGGT-3' \\
RRM2 & F: 5'-CACGGAGCCGAAAACTAAAGC-3' \\
& R: 5'-TCTGCCTTCTTATACATCTGCCA-3' \\
U6 & F: 5'-CTCGCTTCGGCAGCACA-3' \\
& R: 5'-AACGCTTCACGAATTTGCGT-3' \\
\multirow{2}{*}{-actin } & F: 5'-ATTGCCGACAGGATGCAGAA-3' \\
& R: 5'-CAAGATCATTGCTCCTCCTGAGCGCA-3' \\
\hline
\end{tabular}

coated with $50 \mu \mathrm{l}$ of Matrigel matrix (1:5, BD, NJ, USA). A total of $5 \times 10^{4}$ cells were seeded in the upper chamber of a Transwell insert. The specific details were described in a previous report [17].

Luciferase reporter assay. Wild-type (WT) or mutant (MUT) SNHG16 binding sites were subcloned into the pGL3-control vector (Promega, Madison, WI, USA). MDA-MB-231 and MCF-7 cells were seeded into 24-well plates. The miR-384 mimic was co-transfected with wild-type SNHG16 (WT-SNHG16) or mutant SNHG16 (MUT-SNHG16) using Lipofectamine 2000 according to the manufacturer's instructions. Putative miR-30a binding sites in the 3' untranslated region (UTR) of the RRM2 mRNA transcript were synthesized and inserted into the pGL3-control vector. For the reporter assay, MDA-MB-231 and MCF-7 cells were plated into 24-well plates and transfected with wildtype RRM2 (WT-RRM2) or mutant RRM2 (MUT-RRM2) and the miR-30a mimic using Lipofectamine 2000. After transfection for $48 \mathrm{~h}$, the cells were harvested and assayed with a luciferase reporter assay system (Promega, Madison, WI, USA) according to the manufacturer's instructions.

RNA immunoprecipitation (RIP) assay. A RIP assay was performed according to the instructions of the EZ-Magna RIP RNA-Binding Protein Immunoprecipitation Kit (Millipore, Billerica, MA). Cells in different groups were lysed in a lysis buffer containing a protease inhibitor cocktail and an RNase inhibitor. The cells were incubated with a RIP buffer containing magnetic beads coated with anti-Ago 2 antibodies (Millipore). IgG acted as a negative control. After incubation for $2 \mathrm{~h}$ at $4{ }^{\circ} \mathrm{C}$ the co-precipitated RNA was eluted from the beads and measured by PCR analysis.

Western blot analysis. Western blot analysis was performed as described previously [17]. Briefly, total protein was extracted from transfected cells for Western blot analysis. Antibodies against RRM2 (67006-1-Ig, 1:10000) and $\beta$-actin (60008-1-Ig, 1:4000) and a horseradish peroxidase-linked secondary antibody (SA00001-1, 1:5000) were obtained from Proteintech.

Statistical analysis. Statistical analyses were performed using GraphPad software (GraphPad Prism version 5.0, San Diego, USA). The results are expressed as the means \pm SEMs. P-values were analyzed using Student's t test. A p $<0.05$ was considered to indicate a statistically significant difference.

\section{Results}

Tumor cell proliferation and invasion are inhibited by knocking down SNHG16 expression in vitro. First, we examined SNHG16 expression in breast cancer cell lines (MDA-MB-231 and MCF-7). qRT-PCR showed that SNHG16 expression was significantly upregulated in the breast cancer cell lines compared to the MCF-10A cell line (Figure 1A). To investigate the role of SNHG16 in breast cancer cells, si-SNHG16 was transfected into breast cancer cells. The transfection efficiency was confirmed by qRT-PCR analysis (Figure 
1B). The CCK- 8 and colony formation assay results indicated that the downregulation of SNHG16 expression significantly inhibited proliferation in si-SNHG16-transfected breast cancer cells compared to that in si-NC-transfected cells (Figures 1C, 1D). Next, flow cytometry was performed to determine the effect of SNHG16 on cell apoptosis. We found that knocking down SNHG16 expression significantly induced cell apoptosis in breast cancer cells (Figure 1E). To clarify the role of SNHG16 in breast cancer metastasis, the present study analyzed the effects of SNHG16 on the migration and invasion of breast cancer cells. Via Transwell assays, significant decreases in migration and invasion were observed in breast cancer cells transfected with si-SNHG16 compared with cells transfected with si-NC (Figure 1F).

Reciprocal interaction between SNHG16 and miR-30a in breast cancer cells. Recent studies have shown that lncRNAs act as competing endogenous RNAs (ceRNAs) or molecular sponges to modulate the biological roles of miRNAs [19].
To investigate whether SNHG16 interacts with miRNAs, we examined whether SNHG16 possesses a potential binding site for miR-30a using the starBase web tool (Figure 2A). Cheng et al. showed that miR-30a is a tumor suppressor that inhibits breast cancer progression by targeting vimentin [20]. To verify whether SNHG16 binds with miR-30a in breast cancer cells, a dual luciferase reporter assay was conducted and showed that the miR-30a mimic significantly reduced the luciferase activity of WT-SNHG16 in breast cancer cells (Figure 2B). qRT-PCR showed that knocking down SNHG16 expression significantly increased miR-30a expression in breast cancer cells (Figure 2C). In addition, miR-30a expression was significantly decreased in breast cancer cell lines (Figure 2D). Moreover, a RIP assay showed that SNHG16 and miR-30a expression was significantly enriched in the Ago2 pellet compared to the IgG control pellet (Figure 2E). These data indicate that SNHG16 interacts with miR-30a and decreases its expression in breast cancer.
A

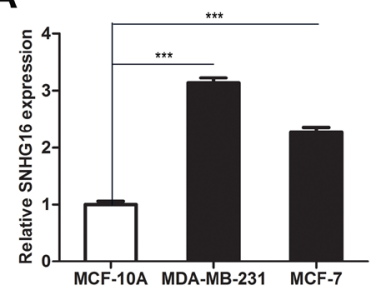

D

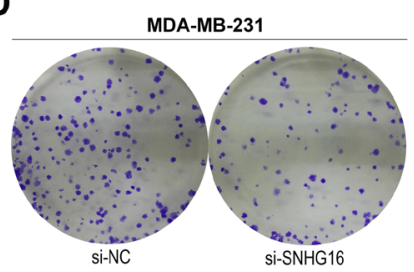

$\mathbf{E}$
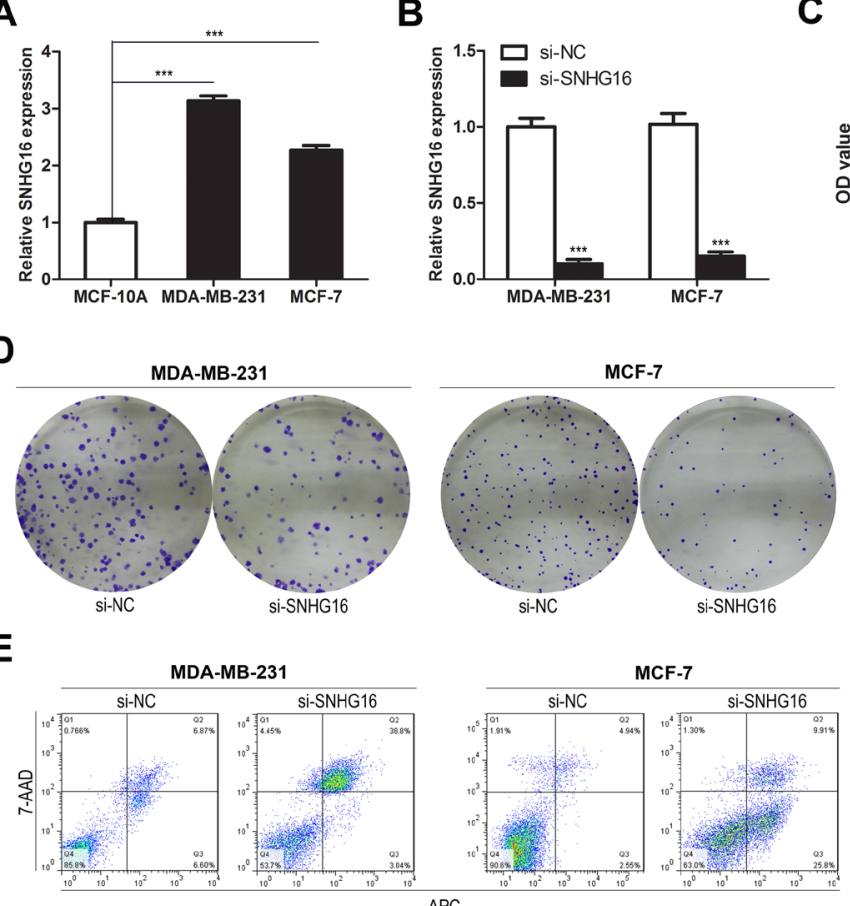

C
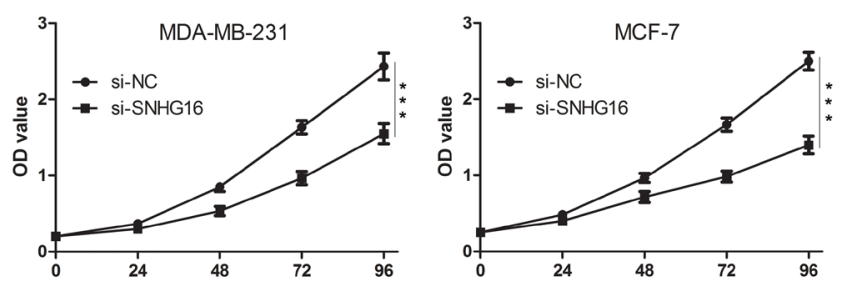
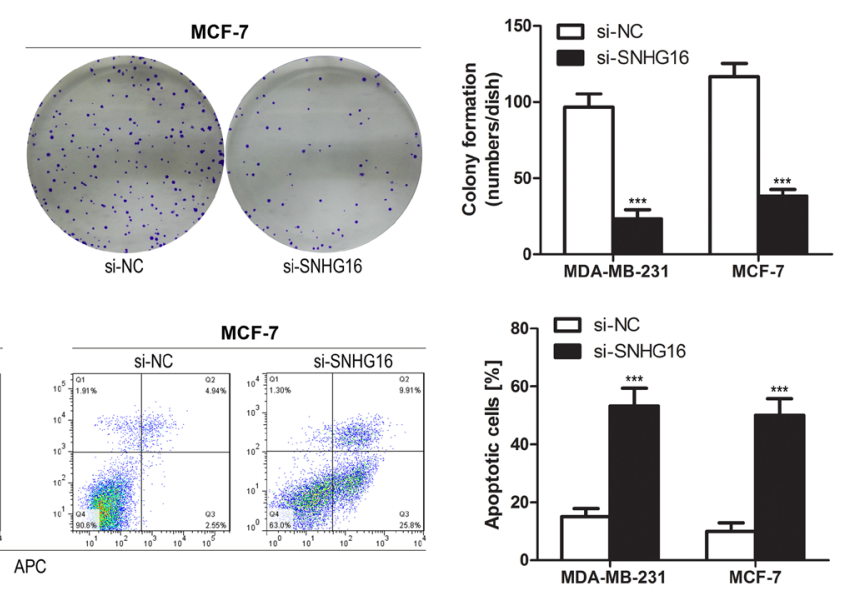

$\mathbf{F}$
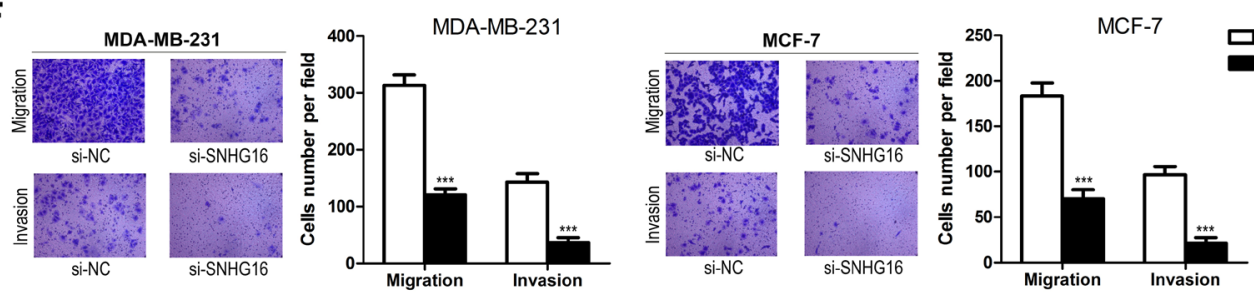

Figure 1. SNHG16 promotes breast cancer cell proliferation and invasion and inhibits apoptosis in vitro. A) qRT-PCR was used to examine SNHG16 expression in breast cancer cell lines and immortalized breast epithelial cells. B) qRT-PCR verified the interference efficiency of si-SNHG16 in breast cancer cell lines. C) CCK-8 assay to determine the effects of SNHG16 on breast cancer cell proliferation. D) Colony formation assay to determine the effects of SNHG16 on the breast cancer cell colony number. E) Flow cytometric analysis of the effects of SNHG16 on breast cancer cell apoptosis. F) Transwell assay to determine the effects of SNHG16 on breast cancer cell migration and invasion. The data are presented as the means \pm SEMs, ${ }^{\star * *}$ p $<0.001$ 
A

WT SNHG16 5' UUUCCUGUUUGUAAGAUUUGUUUACa 3'

5.

miR-30a 3' gaAGGUC-AGC-UCCU-- ACAAAUG 5'

MUT SNHG16 5' UUUCCUGUUUGUAAGAUUACAAAUGa 3
B

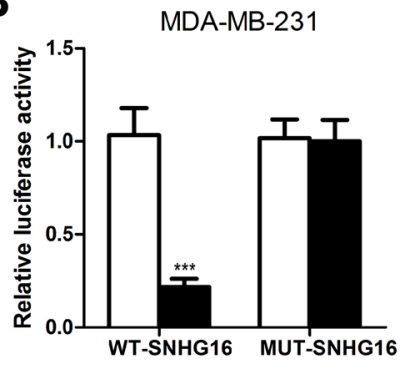

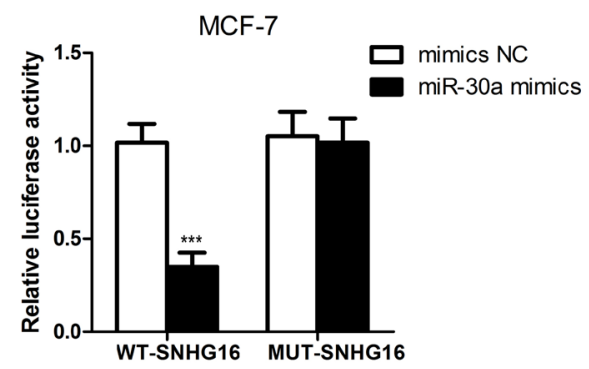

C

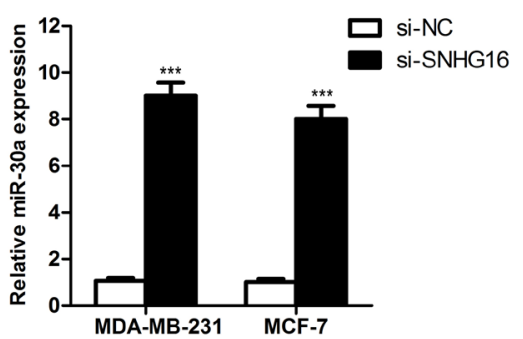

D

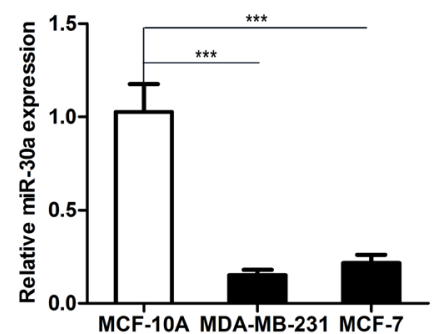

E

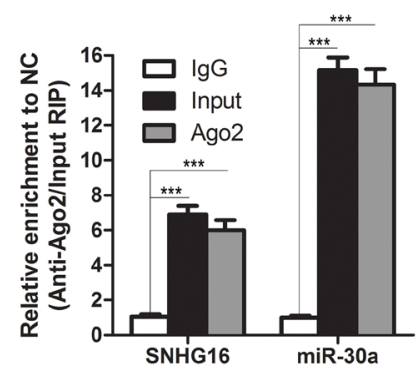

Figure 2. SNHG16 interacts with miR-30a and decreases its expression in breast cancer. A) The predicted position of the miR-30a binding sites in SNHG16 and the mutated sequences of SNHG16 used in the dual luciferase reporter assay. B) The dual luciferase reporter assay showed that the miR30a mimic significantly decreased the luciferase activity of WT-SNHG16 in breast cancer cells. C) SNHG16-mediated inhibition of miR-384 expression in breast cells. D) The expression level of miR-30a in cell lines was measured by qRT-PCR. E) The RIP assay showed that compared to the IgG control, both SNHG16 and miR-30a were enriched in Ago2-containing miRNAs. The data are presented as the means \pm SEMs, ${ }^{\star * *}$ p $<0.001$

Downregulating miR-30a expression abolishes the effects of knocking down SNHG16 expression on breast cancer cells. To further investigate whether miR-30a participates in SNHG16-induced proliferation, apoptosis, migration, and invasion in breast cancer cells, miR-30a expression was downregulated by miR-30a inhibitors in breast cancer cells with knocked down SNHG16 expression (Figure 3A). Knocking down miR-30a expression promoted proliferation and colony formation and inhibited apoptosis in breast cancer cells with knocked down SNHG16 expression (Figures 3B-3D). Moreover, knocking down miR-30a expression reversed the SNHG16 silencing-induced attenuation of migration and invasion by breast cancer cells (Figure 3E). Taken together, these results implied that miR-30a might be involved in SNHG16-mediated oncogenic activity in breast cancer.

miR-30a inhibits breast cancer cell proliferation and invasion by targeting RRM2. Bioinformatic analyses (miRanda) revealed that RRM2 is a potential target of miR-30a (Figure 4A). Previous studies have suggested that RRM2 is an oncogene involved in breast cancer cell proliferation, apoptosis, migration, and invasion [21]. To confirm RRM2 as a direct target of miR-30a, we engineered luciferase reporter constructs containing a WT or MUT 3' UTR of the RRM2 gene. The luciferase reporter assay showed that miR-30a significantly decreased the luciferase activity of the WT RRM2 3' UTR but not that of the MUT RRM2 3' UTR in MDA-MB-231 and MCF-7 cells (Figure 4B). Further- more, western blot analysis revealed that transfection of the miR-30a mimic into MDA-MB-231 and MCF-7 cells led to considerable reductions in the protein levels of RRM2 (Figure 4C). Taken together, these data show that RRM2 is a direct target of miR-30a in breast cancer.

SNHG16/miR-30a axis promotes breast cancer cell proliferation and invasion by regulating RRM2. To test whether SNHG16 can regulate RRM2 via miR-30a in breast cancer cells, a western blot analysis was performed. Transfection with si-SNHG16 significantly decreased RRM2 expression (Figure 5A). Transfection with a miR-30a inhibitor significantly increased RRM2 expression (Figure 5B), and compared with co-transfection with si-SNHG16 and inhibitor NC, co-transfection with si-SNHG16 and the miR-30a inhibitor increased RRM2 expression (Figure 5C).

Breast cancer cell proliferation and invasion are inhibited by knockdown of RRM2 expression in vitro. To evaluate the effects of RRM2 on breast cancer cells, we suppressed RRM2 via siRNA. The transfection efficiency was confirmed by qRT-PCR analysis (Figure 6A). The CCK- 8 and colony formation assay results indicated that the downregulation of RRM2 expression significantly inhibited proliferation in si-RRM2-transfected breast cancer cells compared to the negative control cells (Figures 6B, 6C). The flow cytometric assay results indicated that knockdown of SNHG16 expression significantly induced cell apoptosis in breast cancer cells (Figure 6D). Transwell assays showed significant decreases in the migration and invasion of breast cancer cells transfected 
A

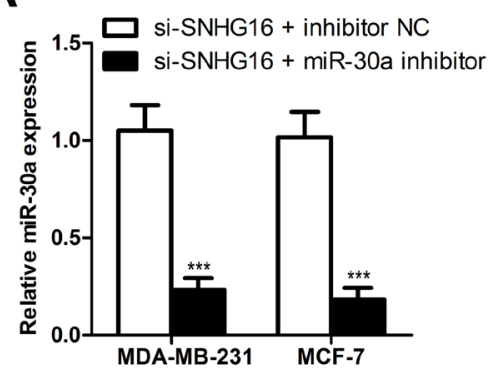

C

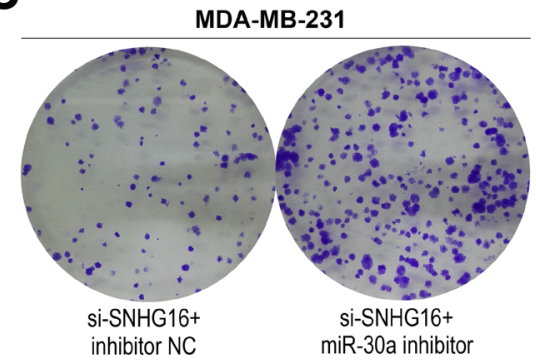

D

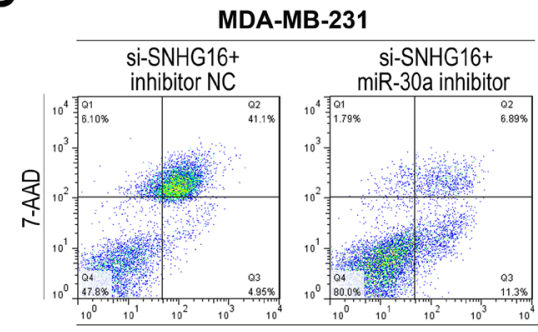

E

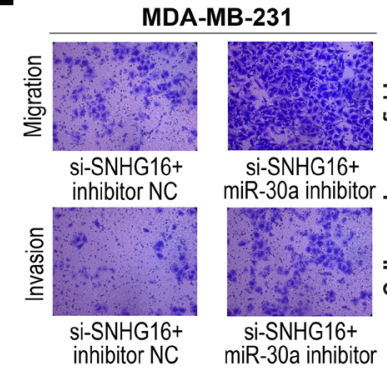

B
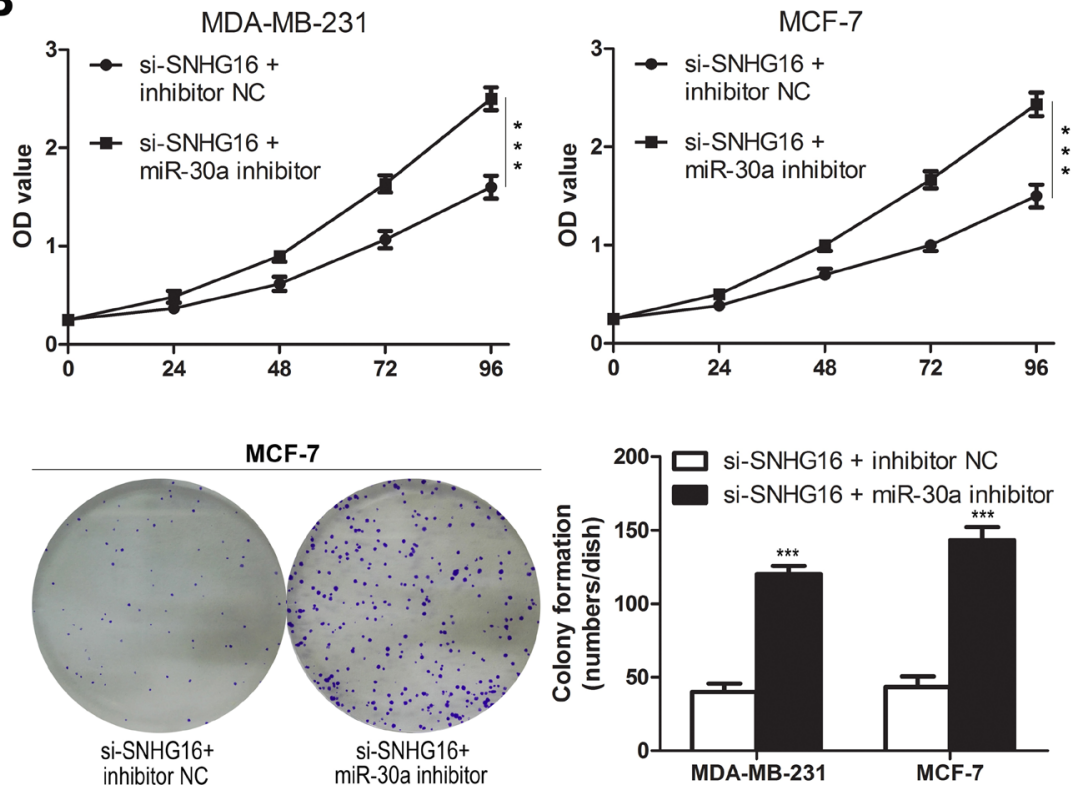
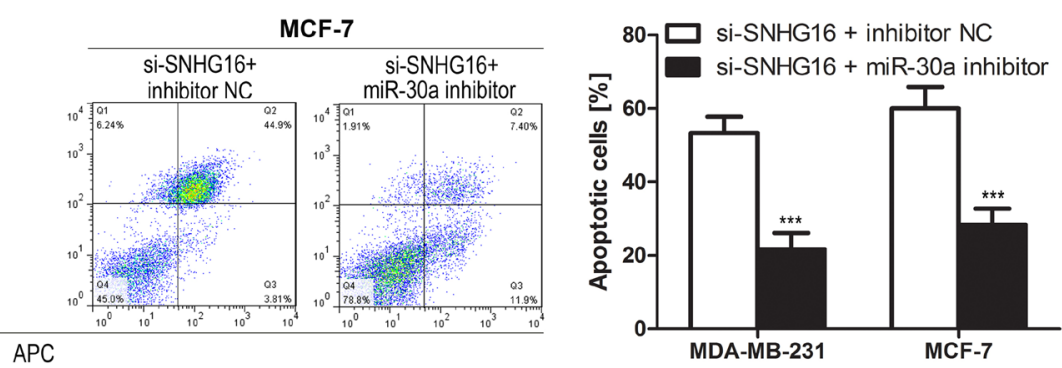

MDA-MB-231
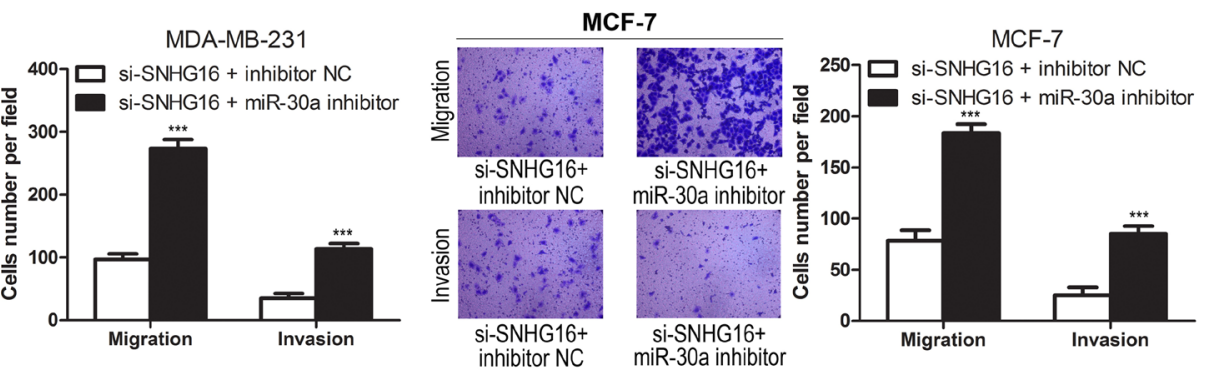

Figure 3. miR-30a reverses the tumor-promoting effects of SNHG16 in breast cancer cells. A) qRT-PCR verified the interference efficiency of miR-30a in breast cancer cells after co-transfection with si-SNHG16 and the miR-30a inhibitor. B) CCK-8 assay to investigate breast cancer cell proliferation after co-transfection with si-SNHG16 and the miR-30a inhibitor. C) Colony formation assay to determine the colony number of breast cancer cells after co-transfection with si-SNHG16 and the miR-30a inhibitor. D) Flow cytometric analysis of breast cancer cell apoptosis after co-transfection with si-SNHG16 and the miR-30a inhibitor. E) Transwell assay to investigate breast cancer cell migration and invasion after co-transfection with si-SNHG16 and the miR-30a inhibitor. The data are presented as the means \pm SEMs, ${ }^{* * *} \mathrm{p}<0.001$

with si-RRM2 compared with cells transfected with si-NC (Figure 6E). These results indicate that RRM2 promotes breast cancer cell proliferation, colony formation, migration, and invasion and inhibits the apoptosis of breast cancer cells. Moreover, our results show that SNHG16 promotes breast cancer progression by regulating the miR-30a/RRM2 axis (Figure 6F).

\section{Discussion}

Although advances in clinical therapies have improved clinical conditions, breast cancer patients with advancedstage disease or distant metastasis still have a very poor prognosis; therefore, new targets limiting breast cancer cell proliferation and metastasis are urgently needed. Currently, 
A

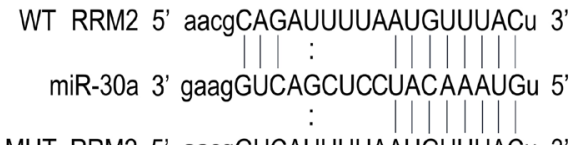

MUT RRM2 5' aacgGUCAUUUUAAUGUUUACu 3'
B

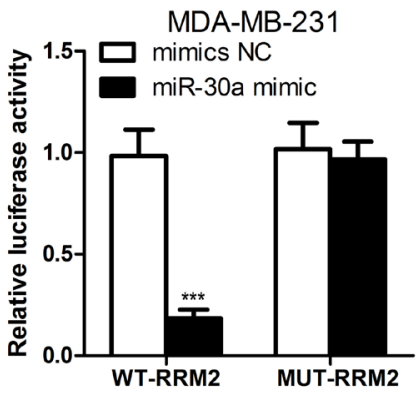

MCF-7

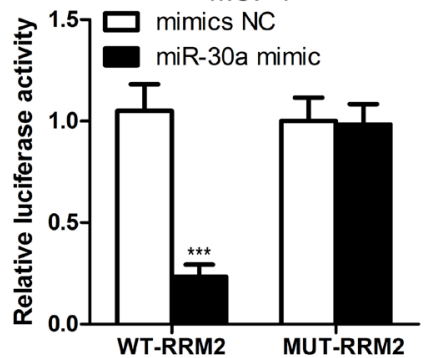

\section{C}
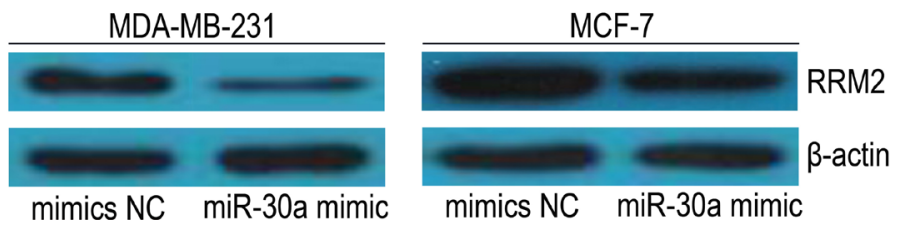

Figure 4. RRM2 is a target gene of miR-30a in breast cancer. A) The predicted position of the miR-30a binding sites in RRM2 and the mutated sequences of RRM2 used in the dual luciferase reporter assay. B) The dual luciferase reporter assay demonstrated that the miR-30a mimic significantly decreased the luciferase activity of WT-RRM2 in breast cancer cells. C) The protein levels of RRM2 were decreased in breast cancer cells transfected with the miR$30 \mathrm{a}$ mimic, as determined by western blotting. The data are presented as the means $\pm \mathrm{SEMs},{ }^{* * *} \mathrm{p}<0.001$

A

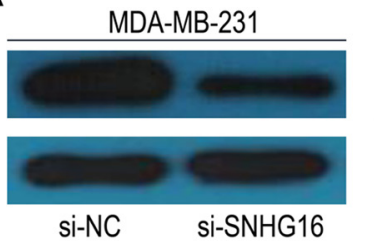

B

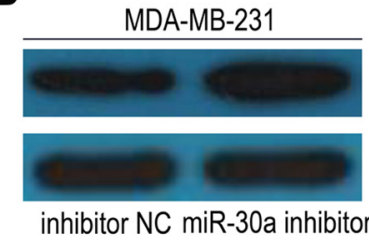

C

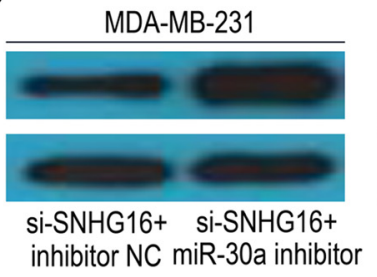

et al. showed that downregulation of the expression of the lncRNA MAGI2-AS3 correlated with tumor progression and poor prognosis in breast cancer [18]. Luan et al. found that the IncRNA MIAT promoted breast cancer progression and functioned as a ceRNA to regulate DUSP7 expression by sponging miR-155-5p [24]. However, the precise molecular mechanisms underlying the progression and development of breast cancer remain largely unclear.

SNHG16 has been identified as a potential oncogene in many cancers [25-30]. However, the function of SNHG16 in breast cancer has not been completely investigated. In this study, our results showed that the downregulation of SNHG16 expression inhibited cancer cell proliferation, migration, and invasion in vitro and retarded tumor growth in vivo. Taken together, these results suggest that SNHG16 acts as an oncogenic lncRNA in breast cancer progression.

miRNAs are short noncoding RNA molecules with lengths of 20-24 nucleotides that play important roles in the posttranscriptional regulation of gene expression [31]. Accumulating evidence indicates that miRNAs play significant roles in the progression of various diseases, including cancers $[32,33]$. Recently, an increasing number of studies have shown that lncRNAs perform their roles by serving as ceRNAs to regulate miRNA expression and functions [34, 35]. To further explore the underlying molecular mechanism by which SNHG16 regulates breast cancer, we performed a bioinformatic prediction analysis and found that miR-30a was a potential candidate. Luciferase reporter activity and RIP assays showed that SNHG16 might act as a molecular sponge for miR-30a in breast cancer progression. Moreover, miR-30a inhibitors abolished the effects of knocking down SNHG16 expression on breast cancer cells. Previous studies 
A

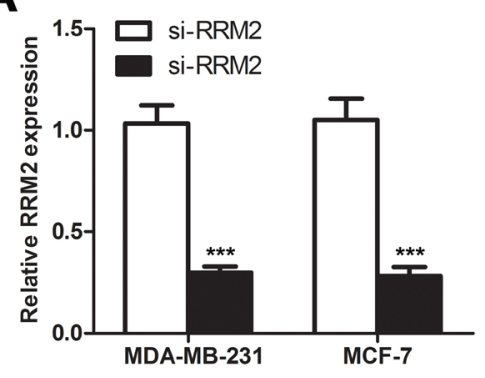

B
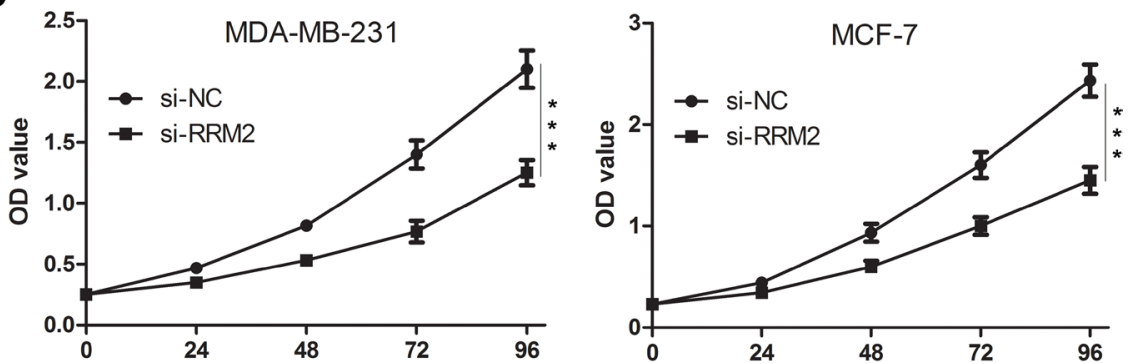

C
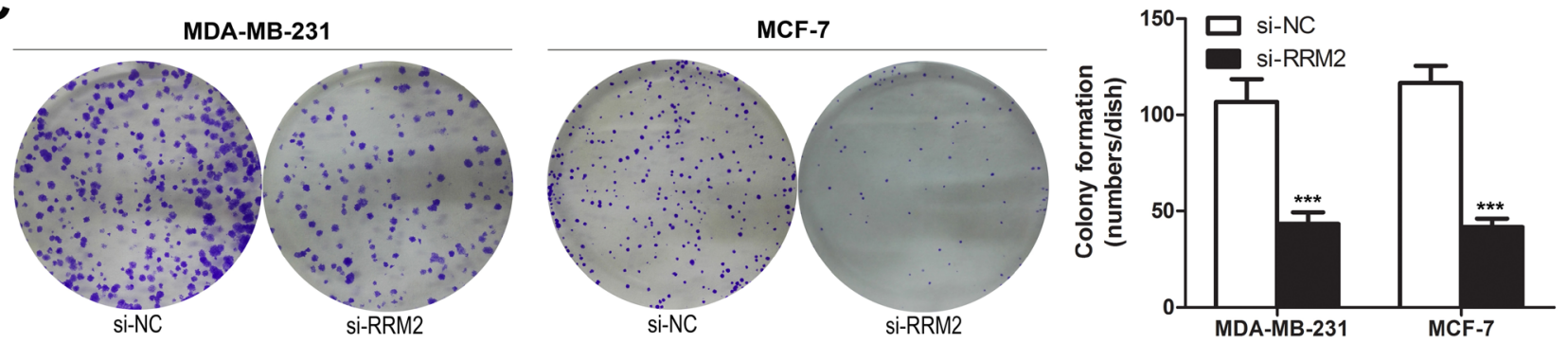

D
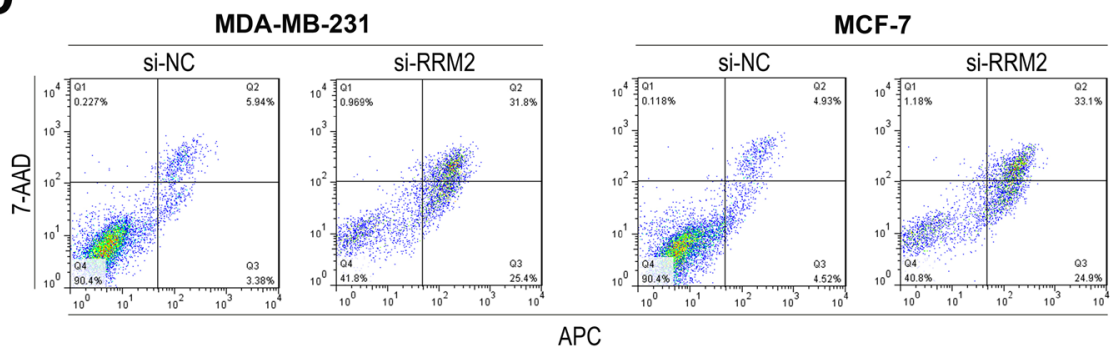

E
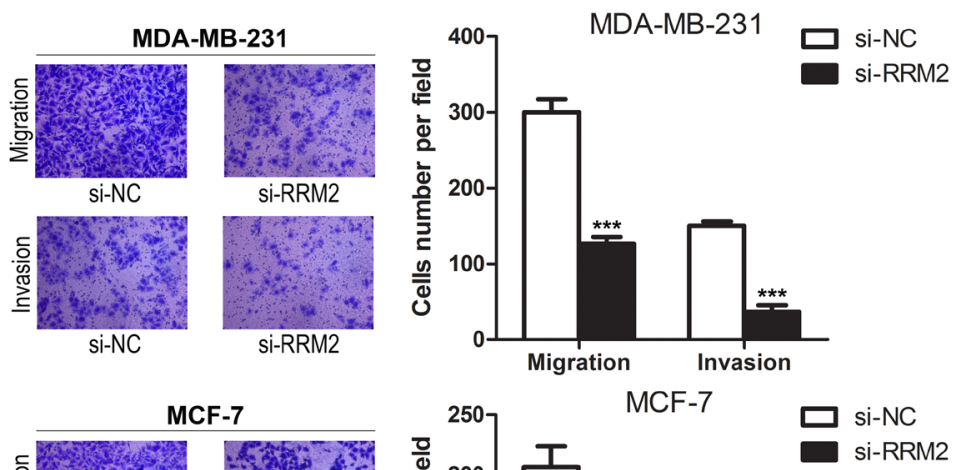

MCF-7

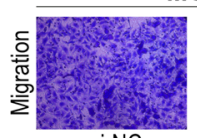

si-NC

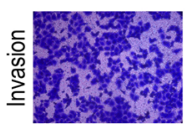

si-NC

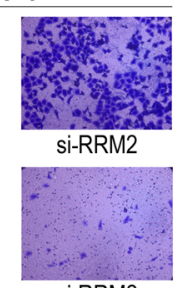

si-RRM2

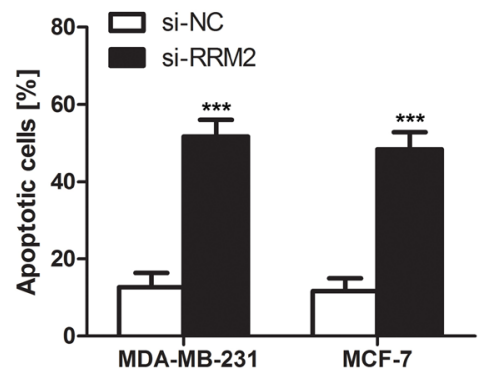

$\mathbf{F}$

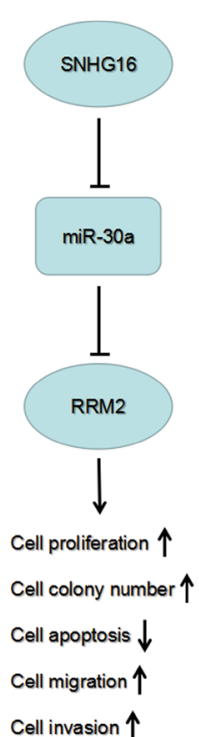

Figure 6. RRM2 promotes breast cancer cell proliferation and invasion and inhibits apoptosis in vitro. A) The interference efficiency of si-RRM2 in breast cancer cell lines was verified by qRT-PCR. B) A CCK-8 assay was conducted to evaluate the effects of RRM2 on breast cancer cell proliferation. C) A colony formation assay was conducted to evaluate the effects of RRM2 on the breast cancer cell colony number. D) Flow cytometric analysis was conducted to assess the effects of RRM2 on breast cancer cell apoptosis. E) A Transwell assay was conducted to evaluate the effects of RRM2 on breast cancer cell migration and invasion. F) Schematic of the mechanism derived from the investigations in this study. The data are presented as the means \pm SEMs, ${ }^{* * *} \mathrm{p}<0.001$ 
have shown that miR-30a acts as a tumor suppressor in breast cancer [20]. Taken together, these results suggest that SNHG16 promotes breast cancer progression through the inhibition of miR-30a.

RRM2 is the catalytic subunit of ribonucleotide reductase; an essential enzyme involved in DNA synthesis and can regulate the enzymatic activity of this reductase [36]. Our data showed that RRM2 inhibition reduced the proliferation and invasion of breast cancer cells in vitro. This result was consistent with that of Putluri et al. [37]. The data in Putluri's report showed that inhibition of RRM2 suppressed tumor growth and decreased cell migration and invasion in breast cancer. In this study, we used online biological software to predict that RRM2 is a potential target gene of miR-30a. Here, we found that the knocking down SNHG16 expression significantly decreased RRM2 expression at the mRNA and protein levels in breast cancer cells. Moreover, compared with co-transfection with si-SNHG16 and inhibitor NC, co-transfection with si-SNHG16 and a miR-30a inhibitor increased RRM2 expression in breast cancer cells. Furthermore, CCK-8, colony formation, and Transwell assays showed that compared with co-transfection with si-SNHG16 and inhibitor NC, co-transfection with si-SNHG16 and the miR-30a inhibitor significantly increased cell proliferation, colony formation, migration, and invasion and induced cell apoptosis in breast cancer cells. These results suggest that SNHG16 exerts its oncogenic function, at least in part, by regulating the miR-33a/RRM2 axis.

In conclusion, our studies demonstrate that the lncRNA SNHG16 performs an oncogenic role in breast cancer progression by functioning as a ceRNA for miR-30a to regulate RRM2. These findings provide a novel mechanism for the occurrence and development of breast cancer.

Acknowledgments: This work was supported by grants from the Zibo key research and development plan (No. 2018kj010140), the Project of Shandong Province Higher Educational Science and Technology Program (No. J18KA299) and the National Natural Science Foundation of China (No. 81602330).

\section{References}

1. SIEGEL RL, MILLER KD, JEMAL A. Cancer Statistics, 2017. CA Cancer J Clin 2017; 67: 7-30. https://doi.org/10.3322/ caac. 21387

2. TAO Z, SHI A, LU C, SONG T, ZHANG Z et al. Breast Cancer: Epidemiology and Etiology. Cell Biochem Biophys 2015; 72: 333-338. https://doi.org/10.1007/s12013-014-0459-6

3. CHOU J, WANG B, ZHENG T, LI X, ZHENG L et al. MALAT1 induced migration and invasion of human breast cancer cells by competitively binding miR-1 with cdc42. Biochem Biophys Res Commun 2016; 472: 262-269. https://doi. org/10.1016/j.bbrc.2016.02.102

4. HANAHAN D, WEINBERG RA. Hallmarks of cancer: the next generation. Cell 2011; 144: 646-674. https://doi. org/10.1016/j.cell.2011.02.013
5. QUINN JJ, CHANG HY. Unique features of long non-coding RNA biogenesis and function. Nat Rev Genet 2016; 17: 47-62. https://doi.org/10.1038/nrg.2015.10

6. MARTENS-UZUNOVA ES, BOTTCHER R, CROCE CM, JENSTER G, VISAKORPI T et al. Long noncoding RNA in prostate, bladder, and kidney cancer. Eur Urol 2014; 65: 1140-1151. https://doi.org/10.1016/j.eururo.2013.12.003

7. CHENG J, CHEN J, ZHANG X, MEI H, WANG $\mathrm{F}$ et al. Overexpression of CRNDE promotes the progression of bladder cancer. Biomed Pharmacother 2018; 99: 638-644. https://doi.org/10.1016/j.biopha.2017.12.055

8. SUN MD, ZHENG YQ, WANG LP, ZHAO HT, YANG S. Long noncoding RNA UCA1 promotes cell proliferation, migration and invasion of human leukemia cells via sponging miR-126. Eur Rev Med Pharmacol Sci 2018; 22: 2233 2245. https://doi.org/10.26355/eurrev_201804_14809

9. DONG MM, PENG SJ, YUAN YN, LUO HP. LncRNA TTN-AS1 contributes to gastric cancer progression by acting as a competing endogenous RNA of miR-376b-3p. Neoplasma 2019; 66: 564-575. https://doi.org/10.4149/ neo_2018_180927N721

10. GAO XF, HE HQ, ZHU XB, XIE SL, CAO Y. LncRNA SNHG20 promotes tumorigenesis and cancer stemness in glioblastoma via activating PI3K/Akt/mTOR signaling pathway. Neoplasma 2019; 66: 532-542. https://doi.org/10.4149/ neo_2018_180829N656

11. GU J, WANG Y, WANG X, ZHOU D, WANG X et al. Effect of the LncRNA GAS5-MiR-23a-ATG3 Axis in Regulating Autophagy in Patients with Breast Cancer. Cell Physiol Biochem 2018; 48: 194-207. https://doi.org/10.1159/000491718

12. ZHANG Y, LI J, JIA S, WANG Y, KANG Y et al. Downregulation of lncRNA-ATB inhibits epithelial-mesenchymal transition of breast cancer cells by increasing miR-141-3p expression. Biochem Cell Biol 2019; 97: 193-200. https://doi. org/10.1139/bcb-2018-0168

13. ZHANG K, CHEN J, SONG H, CHEN LB. SNHG16/miR140-5p axis promotes esophagus cancer cell proliferation, migration and EMT formation through regulating ZEB1. Oncotarget 2017; 9: 1028-1040. https://doi.org/10.18632/ oncotarget. 23178

14. ZHU H, ZENG Y, ZHOU CC, YE W. SNHG16/miR-216$5 \mathrm{p} / \mathrm{ZEB} 1$ signal pathway contributes to the tumorigenesis of cervical cancer cells. Arch Biochem Biophys 2018; 637: 1-8. https://doi.org/10.1016/j.abb.2017.11.003

15. FENG F, CHEN A, HUANG J, XIA Q, CHEN Y et al. Long noncoding RNA SNHG16 contributes to the development of bladder cancer via regulating miR-98/STAT3/Wnt/betacatenin pathway axis. J Cell Biochem 2018; 119: 9408-9418. https://doi.org/10.1002/jcb.27257

16. YANG BY, MENG Q, SUN Y, GAO L, YANG JX. Long noncoding RNA SNHG16 contributes to glioma malignancy by competitively binding miR-20a-5p with E2F1. J Biol Regul Homeost Agents 2018; 32: 251-261.

17. DU S, HU W, ZHAO Y, ZHOU H, WEN W et al. Long noncoding RNA MAGI2-AS3 inhibits breast cancer cell migration and invasion via sponging microRNA-374a. Cancer Biomark 2019; 24: 269-277. https://doi.org/10.3233/CBM182216 
18. YANG Y, YANG H, XU M, ZHANG H, SUN $M$ et al. Long non-coding RNA (lncRNA) MAGI2-AS3 inhibits breast cancer cell growth by targeting the Fas/FasL signalling pathway. Hum Cell 2018; 31: 232-241. https://doi.org/10.1007/ s13577-018-0206-1

19. TAY Y, RINN J, PANDOLFI PP. The multilayered complexity of ceRNA crosstalk and competition. Nature 2014; 505 : 344-352. https://doi.org/10.1038/nature12986

20. CHENG CW, WANG HW, CHANG CW, CHU HW, CHEN $\mathrm{CY}$ et al. MicroRNA-30a inhibits cell migration and invasion by downregulating vimentin expression and is a potential prognostic marker in breast cancer. Breast Cancer Res Treat 2012; 134: 1081-1093. https://doi.org/10.1007/s10549-0122034-4

21. LIANG WH, LI N, YUAN ZQ, QIAN XL, WANG ZH. DSCAM-AS1 promotes tumor growth of breast cancer by reducing miR-204-5p and up-regulating RRM2. Mol Carcinog 2019; 58: 461-473. https://doi.org/10.1002/mc.22941

22. CUI L, DONG Y, WANG X, ZHAO X, KONG C et al. Downregulation of long noncoding RNA SNHG1 inhibits cell proliferation, metastasis, and invasion by suppressing the Notch-1 signaling pathway in pancreatic cancer. J Cell Biochem 2019; 120: 6106-6112. https://doi.org/10.1002/ jcb. 27897

23. LI Y, WU Z, YUAN J, SUN L, LIN L et al. Long non-coding RNA MALAT1 promotes gastric cancer tumorigenicity and metastasis by regulating vasculogenic mimicry and angiogenesis. Cancer Lett 2017; 395: 31-44. https://doi. org/10.1016/j.canlet.2017.02.035

24. LUAN T, ZHANG X, WANG S, SONG Y, ZHOU S et al. Long non-coding RNA MIAT promotes breast cancer progression and functions as ceRNA to regulate DUSP7 expression by sponging miR-155-5p. Oncotarget 2017; 8: 7615376164. https://doi.org/10.18632/oncotarget.19190

25. LI S, ZHANG S, CHEN J. c-Myc induced upregulation of long non-coding RNA SNHG16 enhances progression and carcinogenesis in oral squamous cell carcinoma. Cancer Gene Ther 2019; 26: 400-410. https://doi.org/10.1038/ s41417-018-0072-8

26. ZHU C, CHENG D, QIU X, ZHUANG M, LIU Z. Long Noncoding RNA SNHG16 Promotes Cell Proliferation by Sponging MicroRNA-205 and Upregulating ZEB1 Expression in Osteosarcoma. Cell Physiol Biochem 2018; 51: 429440. https://doi.org/10.1159/000495239
27. HAN W, DU X, LIU M, WANG J, SUN L et al. Increased expression of long non-coding RNA SNHG16 correlates with tumor progression and poor prognosis in non-small cell lung cancer. Int J Biol Macromol 2019; 121: 270-278. https://doi. org/10.1016/j.ijbiomac.2018.10.004

28. YANG XS, WANG GX, LUO L. Long non-coding RNA SNHG16 promotes cell growth and metastasis in ovarian cancer. Eur Rev Med Pharmacol Sci 2018; 22: 616-622. https://doi.org/10.26355/eurrev_201802_14284

29. CAI C, HUO Q, WANG X, CHEN B, YANG Q. SNHG16 contributes to breast cancer cell migration by competitively binding miR-98 with E2F5. Biochem Biophys Res Commun 2017; 485: 272-278. https://doi.org/10.1016/j. bbrc.2017.02.094

30. LI W, XU W, SONG JS, WU T, WANG WX. LncRNA SNHG16 promotes cell proliferation through miR-302a-3p/ FGF19 axis in hepatocellular carcinoma. Neoplasma 2019; 66: 397-404. https://doi.org/10.4149/neo_2018_180720N504

31. ESQUELA-KERSCHER A, SLACK FJ. Oncomirs - microRNAs with a role in cancer. Nat Rev Cancer 2006; 6: 259-269. https://doi.org/10.1038/nrc1840

32. DU S, LI H, SUN X, LI D, YANG Y et al. MicroRNA-124 inhibits cell proliferation and migration by regulating SNAI2 in breast cancer. Oncol Rep 2016; 36: 3259-3266. https://doi. org/10.3892/or.2016.5163

33. LIU K, ZHANG C, LI T, DING Y, TU T et al. Let-7a inhibits growth and migration of breast cancer cells by targeting HMGA1. Int J Oncol 2015; 46: 2526-2534. https://doi. org/10.3892/ijo.2015.2949

34. ULITSKY I. Interactions between short and long noncoding RNAs. FEBS Lett 2018; 592: 2874-2883. https://doi. org/10.1002/1873-3468.13085

35. CHAN JJ, TAY Y. Noncoding RNA: RNA Regulatory Networks in Cancer. Int J Mol Sci 2018; 19: E1310. https://doi. org/10.3390/ijms19051310

36. IWAMOTO K, NAKASHIRO K, TANAKA H, TOKUZEN $\mathrm{N}$, HAMAKAWA $\mathrm{H}$. Ribonucleotide reductase $\mathrm{M} 2$ is a promising molecular target for the treatment of oral squamous cell carcinoma. Int J Oncol 2015; 46: 1971-1977. https://doi.org/10.3892/ijo.2015.2912

37. PUTLURI N, MAITY S, KOMMAGANI R, CREIGHTON CJ, PUTLURI V et al. Pathway-centric integrative analysis identifies RRM2 as a prognostic marker in breast cancer associated with poor survival and tamoxifen resistance. Neoplasia 2014; 16: 390-402. https://doi.org/10.1016/j. neo.2014.05.007 